\title{
THE NUMERICAL RADII OF WEIGHTED SHIFT MATRICES AND OPERATORS
}

\author{
Mao-Ting Chien and Hue-An Sheu
}

\begin{abstract}
Let $A$ be an operator on a separable Hilbert space. The numerical range of $A$ is defined as $W(A)=\{\langle A x, x\rangle:\|x\|=1\}$. It is known that the numerical range of a weighted shift operator is a circular disk. In this paper, we compute and compare the numerical radii of certain weighted shift matrices and operators.
\end{abstract}

Mathematics subject classification (2010): 15A60, 47A12.

Keywords and phrases: Numerical range; numerical radius; weighted shift operator.

\section{REFERENCES}

[1] C. A. Berger and J. G. Stampfli, Mapping theorems for the numerical range, American Journal of Mathematics, 89 (1967), 1047-1055.

[2] M. T. ChiEn AND B. S. TAM, Circularity of the numerical range, Linear algebra and Its Applications, 201 (1994), 113-133.

[3] M. T. ChIEn, On the numerical range of tridiagonal operators, Linear Algebra and Its Applications, 246 (1996), 203-214.

[4] M. T. ChiEN AND H. NAKAZATO, The q-numerical radius of weighted shift operators with periodic weights, Linear Algebra and Its Applications, 422 (2007), 198-218.

[5] M. T. ChIEN AND H. NAKAZATO, The numerical radius of a weighted shift operator with geometric weights, Electronic Journal of Linear Algebra, 18 (2009), 58-63.

[6] K. E. Gustafson And D. K. M. RaO, Numerical range, the Field of Values of Linear Operators and Matrices, Springer, New York, 1997.

[7] M. Marcus AND B. N. ShuRE, The numerical range of certain 0,1-matrices, Linear and Multilinear Algebra, 7 (1979), 111-120.

[8] A. L. SHEILDS, Weighted shift operators and analytic function theory, Mathematical Surveys. Vol. 13, American Mathematical Society, Providence, 1974.

[9] Q. F. Stout, The numerical range of a weighted shift, Proceedings of the American Mathematical Society, 88 (1983), 495-502. 